OPEN ACCESS

Conductance of a quantum wire with an attractive smooth impurity potential

To cite this article: Vassilios Vargiamidis and Hariton M Polatoglou 2005 J. Phys.: Conf. Ser. 1089

View the article online for updates and enhancements.
You may also like

- $\frac{\text { The bosenova and axiverse }}{\text { Hirotaka Yoshino and Hideo Kodama }}$
- Quasibound states of the Green's function
$\frac{\text { for weakly quantized systems }}{\text { Curt A Moyer }}$
- Quasibound states and transport
$\frac{\text { characteristics of Au chains with a }}{\text { substitutional S impurity }}$
M Wawrzyniak-Adamczewska and T
Kostyrko

The bosenova and axiverse

Quasibound states of the Green's function for weakly quantized systems

Quasibound states and transport substitutional S impurity Kostyrko
The Electrochemical Society

Advancing solid state \& electrochemical science \& technology

243rd Meeting with SOFC-XVIII

Boston, MA • May 28 - June 2, 2023

Accelerate scientific discovery!
Learn More \& Register

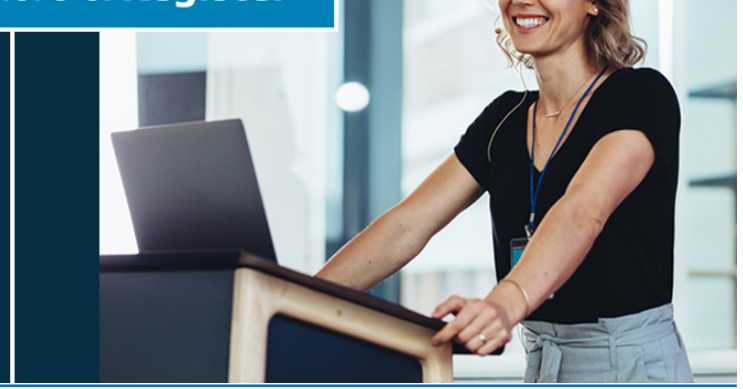

This content was downloaded from IP address 54.197.130.99 on 26/04/2023 at 02:13 


\title{
Conductance of a quantum wire with an attractive smooth impurity potential
}

\author{
Vassilios Vargiamidis and Hariton M Polatoglou \\ Department of Physics, Aristotle University, GR-54124 Thessaloniki, Greece
}

\begin{abstract}
We calculate the conductance through an attractive smoothly varying impurity potential in a quantum wire using the Lippmann-Schwinger equation. The impurity has a decay length along the propagation direction while it is localized along the transverse direction. It is shown that the conductance versus Fermi energy exhibits multiple resonant dips which are due to the creation of quasibound states and their interaction with the continuum states. By varying the size of the impurity the quasibound states evolve into highly localized states with enhanced lifetime.
\end{abstract}

\section{Introduction}

Since the discovery of the quantized conductance [1] in two-dimensional (2D) electron gas microconstrictions, much effort has been devoted to the study of electron scattering from impurities in narrow ballistic channels [2]. In most of these calculations the impurity potential is taken to be the idealized model of the Dirac $\delta$ function. This impurity model is used mainly for two reasons. First, it allows for an analytical solution of the scattering problem with a relatively small amount of effort and second it captures the basic physics of the problem under consideration. However a $\delta$ function impurity in an infinite uniform quantum wire causes some problems. One such problem is the divergence of the quasibound states [3] as the number of transverse modes increases. In addition a $\delta$ function impurity is quite rough whereas any realistic model of an impurity should have a smooth potential profile and finite range.

The purpose of this paper is first to present a brief description of our numerical method for solving the Lippmann-Schwinger (LSE) [4] for an arbitrary shape of the impurity potential and then to apply this method in the case of a smoothly varying attractive impurity with a decay length $\alpha^{-1}$ along the propagation direction. We show that the conductance versus Fermi energy exhibits multiple resonant dips which are due to the formation of discrete levels in the continuum and their interaction with the continuum states. We also investigate the behavior of the resonance width $\Gamma$ and show that increasing the value of $\alpha^{-1}$ leads to a decaying oscillatory behavior of $\Gamma$ while for some suitable values of $\alpha^{-1}$ the width shrinks to infinitesimally small values (which correspond to very stable states with greatly enhanced lifetimes).

\section{Theoretical formulation}

We consider an infinitely long $2 \mathrm{D}$ rectilinear quantum wire in which electrons are confined along the $y$ direction (transverse direction) but are free to move along the $x$ direction (propagation 
direction). The cross section is uniform along the wire. The Hamiltonian can be written as

$$
H=-\frac{\hbar^{2}}{2 m^{*}}+V_{c}(y)+V_{i}(x, y)
$$

where $V_{i}(x, y)$ is the scattering potential of any defects or impurities in the wire, $V_{c}(y)$ is the confining potential and $m^{*}$ is the effective mass of the electron. The energy eigenstates of the unperturbed wire [i.e., when $V_{i}(x, y)=0$ ] are given as $\psi_{\mathbf{p}}^{(0)}(x, y)=e^{i p_{x} x / \hbar} \phi_{n}(y)$, where $\phi_{n}(y)$ are the confinement modes of the wire and the energy eigenvalues are $E=\left(p_{x}^{2} / 2 m^{*}\right)+E_{n}$, where $p_{x}=\hbar k_{x}$ has a continuous spectrum, $n$ are the subband indices and $E_{n}$ define the bottoms of the energy subbands. In the presence of the scattering potential $V_{i}(x, y)$ we have to solve the Schrödinger equation $H|\psi\rangle=E|\psi\rangle$. The solution to this Schrödinger equation takes the form

$$
\psi_{\mathbf{p}}^{(+)}(x, y)=\psi_{\mathbf{p}}^{(0)}(x, y)+\frac{2 m}{\hbar^{2}} \int_{-\infty}^{\infty} d x^{\prime} \int_{-\infty}^{\infty} d y^{\prime} G^{(0)}\left(x, y ; x^{\prime}, y^{\prime}\right) V_{i}\left(x^{\prime}, y^{\prime}\right) \psi_{\mathbf{p}}^{(+)}\left(x^{\prime}, y^{\prime}\right)
$$

which is the LSE of scattering theory written in the position basis. In Eq. (2) $G^{(0)}\left(x, y ; x^{\prime}, y^{\prime}\right)$ is the retarded Green's function of the unperturbed wire and takes the form [5]

$$
G^{(0)}\left(x, y ; x^{\prime}, y^{\prime}\right)=\sum_{n^{\prime}=1}^{\infty} \phi_{n^{\prime}}(y) \phi_{n^{\prime}}^{*}\left(y^{\prime}\right) \frac{e^{i k_{n^{\prime}}\left|x-x^{\prime}\right|}}{2 i k_{n^{\prime}}}
$$

For the propagating modes the wave vectors are $k_{n^{\prime}}=\left[2 m\left(E-E_{n^{\prime}}\right)\right]^{1 / 2} / \hbar$ while for the evanescent modes we set $k_{n^{\prime}}=i \kappa_{n^{\prime}}$. In the Green's function of Eq. (3) the modes $n^{\prime}$ are propagating or evanescent depending on whether $E_{n^{\prime}}$ is less or greater than the Fermi energy $E$.

For a particular form of the potential $V_{i}$, the solution to Eq. (2) for $\psi_{\mathbf{p}}^{(+)}(x, y)$ requires first finding the wave function in the scattering region, i.e., in the region where $V_{i}(x, y) \neq 0$. We consider scattering from an impurity potential of the form $V_{i}(x, y)=\left(\hbar^{2} \gamma / 2 m^{*}\right) \delta\left(y-y_{i}\right) v(x)$, where $v(x)$ is an arbitrary function of the coordinate $x, y_{i}$ is the transversal position of the impurity and $\gamma$ sets the magnitude of the impurity. We also need the transverse energy levels $E_{n, s}$ (i.e, the bottoms of the subbands in the scattering region). These levels are obtained from

$$
\sin (\beta w)=\frac{\gamma}{\beta} \sin \left(\beta y_{i}\right) \sin \left[\beta\left(y_{i}-w\right)\right]
$$

as derived from the Schrödinger equation in the transverse direction, where $\beta=(2 m E)^{1 / 2} / \hbar$ and $w$ is the width of the wire. For the impurity potential given above the LSE Eq. (2) is

$$
\psi_{\mathbf{p}}^{(+)}(x, y)=\psi_{\mathbf{p}}^{(0)}(x, y)+\gamma \int_{-\infty}^{\infty} d x^{\prime} G^{(0)}\left(x, y ; x^{\prime}, y_{i}\right) v\left(x^{\prime}\right) \psi_{\mathbf{p}}^{(+)}\left(x^{\prime}, y_{i}\right)
$$

In order to find to find the wave function $\psi_{\mathbf{p}}^{(+)}(x, y)$ throughout the wire we must know $\psi_{\mathbf{p}}^{(+)}\left(x^{\prime}, y_{i}\right)$ in the region where $v\left(x^{\prime}\right) \neq 0$. Thus our first goal is to find $\psi_{\mathbf{p}}^{(+)}\left(x^{\prime}, y_{i}\right)$.

We assume that the scattering potential extends from $-x_{0}$ to $x_{0}$, that is $v\left(x^{\prime}\right) \neq 0$ for $\left|x^{\prime}\right| \leq x_{0}$ and $v\left(x^{\prime}\right)=0$ for $\left|x^{\prime}\right|>x_{0}$. We divide the interval $\left[-x_{0}, x_{0}\right]$ into $s$ equal subintervals of width $b=2\left|x_{0}\right| / s$ and the coordinates $x^{\prime}$ and $x$ of Eq. (5) are discretized according to $x^{\prime}=-x_{0}+q b$, $x=-x_{0}+r b$, where $q, r=0,1,2, \ldots, s$. The number $s$ of subintervals can be chosen sufficiently large such that the results converge. Replacing now the integral by a sum and setting $y=y_{i}$ in Eq. (5) we obtain a set of $s$ equations (one for each value of $r$ ) for the $s$ unknown values of the wave function $\psi_{\mathbf{p}}^{(+)}$which can be written compactly in matrix form as

$$
\sum_{q=0}^{s} M_{r q} \psi_{q}^{(+)}=-\frac{1}{\gamma b} \psi_{r}^{(0)}
$$


where $M_{r q}=G^{(0)}\left(-x_{0}+r b, y_{i} ;-x_{0}+q b, y_{i}\right) v\left(-x_{0}+q b\right)-(1 / \gamma b) \delta_{r q}$ are the entries of an $s \times s$ matrix which depend also on the electron energy. In the matrix elements $M_{r q}$ the factor $\psi_{q}^{(+)}$ represents the unknown values of the wave function $\psi_{\mathbf{p}}^{(+)}\left(-x_{0}+q b, y_{i}\right)$ in the scattering region and is a column vector. Similarly, $\psi_{r}^{(0)}$ is a column vector and represents the known values of the wave function $\psi_{\mathbf{p}}^{(0)}\left(-x_{0}+r b, y_{i}\right)$ of the incident wave in the region of the impurity. Inverting the matrix $[\mathbf{M}]$ allows us to find $\psi_{q}^{(+)}$(i.e., find the wave function in the scattering region), which was our primary goal. Having found $\psi_{\mathbf{p}}^{(+)}\left(x, y_{i}\right)$ we can now perform the integral in Eq. (5) by discretization (in line with the above discussion) which will allow us to determine $\psi_{\mathbf{p}}^{(+)}\left(x, y_{i}\right)$. The amplitudes $t_{n n^{\prime}}$ are then extracted from the wave function, where $n$ and $n^{\prime}$ are the incident and transmitted modes respectively. The conductance is calculated with Landauer's formula [6]

$$
G=\frac{2 e^{2}}{h} \sum_{n, n^{\prime}} \frac{k_{n^{\prime}}}{k_{n}} t_{n n^{\prime}} t_{n n^{\prime}}^{*}
$$

where $n$ and $n^{\prime}$ run only over the propagating modes of the wire.

\section{Results and discussion}

In this section we present results for the effects of an attractive (smoothly varying) impurity potential on the conductance. In particular for the impurity potential we will employ the model

$$
V_{i}(x, y)=\frac{\hbar^{2} \gamma}{2 m^{*}} \delta\left(y-y_{i}\right) \operatorname{sech}^{2}(\alpha x)
$$

where $\gamma<0$ and $\alpha^{-1}$ is the decay length. The laterally confining potential of the wire is chosen to be an infinite square well. The electron mass is taken to be the effective mass for GaAs which is 0.067 of the free-electron mass. In the numerical calculations we include a total of six modes. In the clean part of the wire the confining potential $V_{c}(y)$ gives rise to the transverse energy levels $E_{n}=\hbar^{2} \pi^{2} n^{2} / 2 m^{*} w^{2}$. We will express all distances in units of the wire width $w$ and all energies in units of $E_{1}$. We also choose $\left|x_{0}\right|=5 \alpha^{-1}$ which is sufficient to guarantee that $V_{i}\left( \pm x_{0}, y\right)=0$. The transverse location of the impurity is at $y_{i}=(5 / 12) w$ so that the first subband in the scattering region opens at $E_{1, s}=-0.53 E_{1}$, the second at $E_{2, s}=3.18 E_{1}$, and the third at $E_{3, s}=6.97 E_{1}$. In Fig. 1 we show the conductance plotted versus the Fermi energy, for three different values of the decay length $\alpha^{-1}$ and the upper two curves are vertically shifted by $1.1 \times\left(2 e^{2} / h\right)$ and $2.2 \times\left(2 e^{2} / h\right)$ respectively for clarity. We notice that the conductance exhibits multiple dips in the first and second subbands and their number increases with increasing values of $\alpha^{-1}$. The dips in the conductance are explained in terms of the formation of quasibound (resonant) states - at special energies - in the impurity region. Electrons at those energies spend enhanced periods of time in the impurity region (i.e., they become temporarily trapped in the quasibound states) and destructive interference of these states with the continuum states causes the conductance to drop resulting in a complete interference blockade of the electron transport.

An important quantity related to resonant scattering is the sharpness of the resonance which is quantified by the width $\Gamma$. In fact since the resonances are only possible at positive collision energies $E_{\text {res }}$, the corresponding quasibound state energies $E_{q b}$ are situated below the positive real axis of the complex $E$ plane, i.e., $E_{q b}=E_{r e s}-i \Gamma$. The width $\Gamma$ determines the lifetime $\tau$ of the quasibound state through the relation $\tau=\hbar / \Gamma$. In Fig. 2 it is seen that the width versus $\alpha^{-1} / w$ first increases, reaches a maximum at $\alpha^{-1}=0.322 w\left(\right.$ where $\Gamma_{\max }=0.1243 E_{1}$ ) and then decreases to a minimum at $\alpha^{-1}=0.4349 w$ (where $\Gamma_{\min } \approx 10^{-6} E_{1}$ ) after which it grows again. This situation can be repeated a few times. The extremely narrow width of the resonant dip at $\alpha^{-1}=0.4349 w$ corresponds to a very stable state - a state with greatly enhanced 


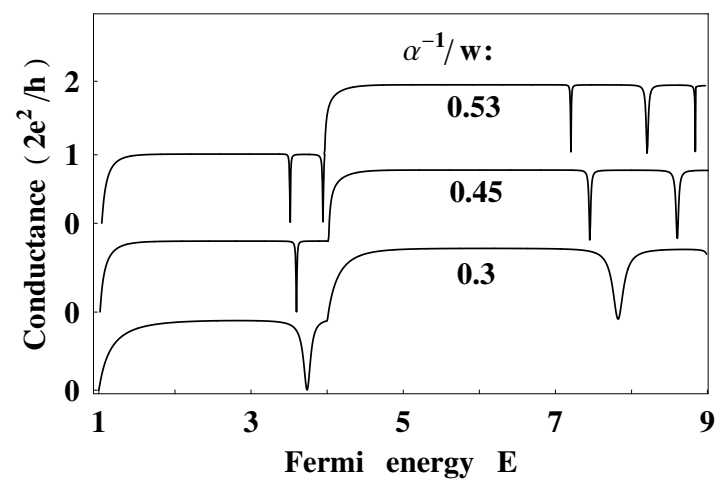

Figure 1. Conductance $G$ (in units of $\left.2 e^{2} / h\right)$ vs $E$ through an impurity $V_{i}(x, y)=$ $\left(\hbar^{2} \gamma / 2 m^{*}\right) \delta\left(y-y_{i}\right) \operatorname{sech}^{2}(\alpha x)$ in a quantum wire of width $w$, and $\gamma=-2.3 \times 10^{6} \mathrm{~cm}^{-1}$.

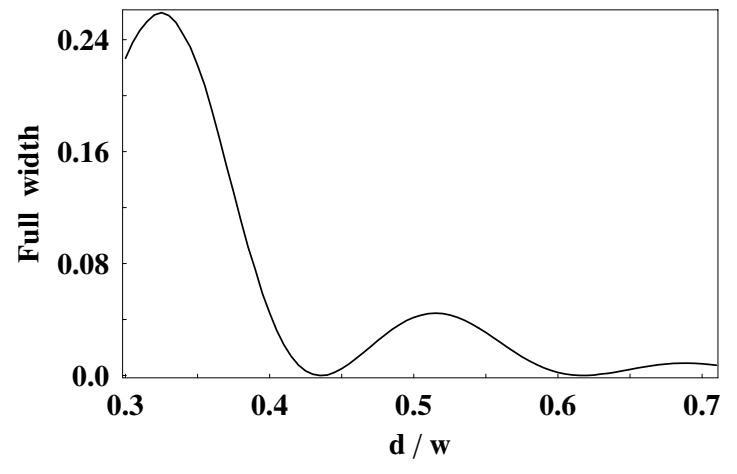

Figure 2. Resonance full width $\Gamma$ (in units of $\left.E_{1}\right)$ vs $\alpha^{-1} / w$. The two minima of the Full width correspond to highly localized states with enhanced lifetimes.

lifetime. Physically this highly localized state is stable since at this particular value of $\alpha^{-1}$ its interaction with the continuum is minimized and therefore it decays extremely slowly (i.e., the decay rate of the localized state into a propagating state is lowest). Thus at this special value of $\alpha^{-1}$ transformation of the quasibound state to a highly localized state occurs.

The increase of the degree of localization of the quasibound states as $\Gamma \rightarrow 0$ can be explained in terms of the coherent interaction of interfering channels in the impurity region. This can be understood if we consider the energy interval $E_{2, s}<E<E_{2}$ where there are two propagating modes $n=1,2$ in the impurity region while outside this region and for the same energy interval only mode $n=1$ is propagating while mode $n=2$ turns into an evanescent wave. Accordingly the coherent resonant interaction of these channels leads to an increase of the charge density in the region of localization and the electron escape rate is minimum.

\section{Summary}

We have considered the transmission of electrons through a smoothly varying attractive impurity with a decay length $\alpha^{-1}$ along the propagation direction in a $2 \mathrm{D}$ rectilinear quantum wire. We have solved the LSE and we showed that the conductance exhibits multiple resonant dips which are due to the creation of quasibound states. In addition we showed that the width of the resonant dip decays in an oscillatory manner and for some critical values of $\alpha^{-1} \Gamma \rightarrow 0$.

\section{References}

[1] van Wees B J, van Houten H, Beenakker C W J, Williamson J G, Kouwenhoven L P, van der Marel D and Foxon C T 1988 Phys. Rev. Lett. 60 848; Wharam D A, Thornton T J, Newbury R, Pepper M, Ahmed H, Frost J E F, Hasko D G, Peacock D C, Ritchie D A and Jones G A C 1988 J. Phys. C 21 L209

[2] Szafer A and Stone A D 1989 Phys. Rev. Lett. 62 300; Chu C S and Sorbello R S 1989 Phys. Rev. B 405941 ; Bagwell P F 1990 Phys. Rev. B 4110 354; Tekman E and Ciraci S 1991 Phys. Rev. B 43 7145; Tekman E and Bagwell P F 1993 Phys. Rev. B 48 2553; Kunze C, Chang L F and Bagwell P F 1996 Phys. Rev. B 53 10 171; Kim C S, Satanin A M, Joe Y S and Cosby R M 1999 Phys. Rev. B 6010 962; Vargiamidis V and Polatoglou H M 2002 Phys. Rev. B 67245303

[3] Boese D, Lischka M and Reichl L E 2000 Phys. Rev. B 61 5632; [24] Boese D, Lischka M and L. E. Reichl L E 2000 Phys. Rev. B 6216933

[4] Sakurai J J 1994 Modern Quantum Mechanics (New York: Addison) chapter 7

[5] Morse P M and Feshbach H 1953 Methods of Theoretical Physics vol 1 (New York: McGraw-Hill)

[6] Buttiker M, Imry Y, Landauer R and Pinhas S 1985 Phys. Rev. B 31 6207; Landauer R 1989 J. Phys. Condens. Matter 18099 